\title{
Effect of Transverse Magnetic Field on Arc Characteristics and Droplet Transfer During Laser- mig Hybrid Welding of Ti-6Al-4V
}

Chen Yue

Huazhong University of Science and Technology

Annan Yin ( $\square$ annan_yin@hust.edu.cn )

The State Key Laboratory of Digital Manufacturing Equipment and Technology, School of Mechanical

Science and Engineering, Huazhong University of Science \& Technology, Wuhan, P. R. China

Dehua Huang

The State Key Laboratory of Digital Manufacturing Equipment and Technology, School of Mechanical Science and Engineering, Huazhong University of Science \& Technology, Wuhan, P. R. China

\section{Research Article}

Keywords: Transverse magnetic field, Laser-MIG hybrid welding, Ti-6Al-4V, Droplet transfer, Arc characteristics

Posted Date: March 19th, 2021

DOI: https://doi.org/10.21203/rs.3.rs-324701/v1

License: (c) (1) This work is licensed under a Creative Commons Attribution 4.0 International License. Read Full License 


\section{Title}

Effect of transverse magnetic field on arc characteristics and droplet transfer during laserMIG hybrid welding of Ti-6Al-4V

\section{Authors and email addresses:}

The first author: Chen Yue

Affiliations: The State Key Laboratory of Digital Manufacturing Equipment and Technology, School of Mechanical Science and Engineering, Huazhong University of Science \& Technology, Wuhan, P. R. China

Postcode: 430074

Telephone: $+86-027-87557742$

Fax numbers: +86-027-87543074

Email addresses: yue30008@,126.com

The second author (corresponding author): Annan Yin

Affiliations: The State Key Laboratory of Digital Manufacturing Equipment and Technology, School of Mechanical Science and Engineering, Huazhong University of Science \& Technology, Wuhan, P. R. China

Postcode: 430074

Telephone: +86-027-87557742

Fax numbers: +86-027-87543074

Email addresses: annan yin@hust.edu.cn 
The third author: Dehua Huang

Affiliations: The State Key Laboratory of Digital Manufacturing Equipment and Technology, School of Mechanical Science and Engineering, Huazhong University of Science \& Technology, Wuhan, P. R. China

Postcode: 430074

Telephone: +86-027-87557742

Fax numbers: +86-027-87543074

Email addresses: huangdehua@hust.edu.cn

\begin{abstract}
In this study, the effect of the transverse magnetic field on the arc characteristics and droplet transfer behavior is investigated during Laser-MIG hybrid welding of Ti-6Al-4V. Especially, transverse magnetic fields with $0 \mathrm{mT}, 8 \mathrm{mT}, 16 \mathrm{mT}, 24$ $\mathrm{mT}$, and $32 \mathrm{mT}$ are studied. Results indicate that an appropriate magnetic field can increase the stability of arc characteristics, improve the droplet detachment efficiency, and reduce the welding defects such as incomplete fusion and undercut. By applying 24 mT transverse magnetic field, the maximum arc area can decrease by $48.7 \%$ with its variance changing from $2.81 \mathrm{~mm}^{2}$ to $1.06 \mathrm{~mm}^{2}$, indicating that an appropriate transverse magnetic field can shrink the arc and improve its stability. The reason of arc shrinkage is that the electric streamline in the arc rotates away from the laser side to the droplet side due to the influence of external magnetic field. On the other hand, the droplet transfer process become more uniform under the appropriate magnetic field. This phenomenon is mainly attributed to the change of Lorentz force direction during droplet rotation, which resultantly increases effective detachment energy. This phenomenon leads to the reduction of the contact time between droplet and molten pool. The droplet transfer form
\end{abstract}


changes from short-circuit transfer to meso-spray transfer under $24 \mathrm{mT}$ magnetic field because of the reduction of the contact time. Therefore, the incomplete fusion and undercut disappears. At last, the appropriated magnetic field parameters during the laserMIG parameters $(2 \mathrm{~kW}, 160 \mathrm{~A}, 2 \mathrm{~m} / \mathrm{min})$ is concluded as $\mathrm{B}=24 \mathrm{mT}$.

Keywords: Transverse magnetic field $\cdot$ Laser-MIG hybrid welding $\cdot$ Ti-6Al-4V·Droplet transfer· Arc characteristics 


\title{
Effect of transverse magnetic field on arc characteristics and droplet transfer during laser-MIG hybrid welding of Ti-6Al-4V
}

\section{Chen Yue ${ }^{1} \cdot$ Annan Yin $^{1} \cdot$ Dehua Huang $^{1}$}

\begin{abstract}
In this study, the effect of the transverse magnetic field on the arc characteristics and droplet transfer behavior is investigated during Laser-MIG hybrid welding of Ti-6Al-4V. Especially, transverse magnetic fields with $0 \mathrm{mT}, 8 \mathrm{mT}, 16 \mathrm{mT}, 24$ $\mathrm{mT}$, and $32 \mathrm{mT}$ are studied. Results indicate that an appropriate magnetic field can increase the stability of arc characteristics, improve the droplet detachment efficiency, and reduce the welding defects such as incomplete fusion and undercut. By applying 24 mT transverse magnetic field, the maximum arc area can decrease by $48.7 \%$ with its variance changing from $2.81 \mathrm{~mm}^{2}$ to $1.06 \mathrm{~mm}^{2}$, indicating that an appropriate transverse magnetic field can shrink the arc and improve its stability. The reason of arc shrinkage is that the electric streamline in the arc rotates away from the laser side to the droplet side due to the influence of external magnetic field. On the other hand, the droplet transfer process become more uniform under the appropriate magnetic field. This phenomenon is mainly attributed to the change of Lorentz force direction during droplet rotation, which
\end{abstract}

\section{$\triangle$ Chen Yue}

yue30008@126.com

\footnotetext{
${ }^{1}$ The State Key Laboratory of Digital Manufacturing Equipment and Technology, School of Mechanical Science and Engineering, Huazhong University of Science \& Technology, 430074 Wuhan, P.R. China
} 
resultantly increases effective detachment energy. This phenomenon leads to the reduction of the contact time between droplet and molten pool. The droplet transfer form changes from short-circuit transfer to meso-spray transfer under $24 \mathrm{mT}$ magnetic field because of the reduction of the contact time. Therefore, the incomplete fusion and undercut disappears. At last, the appropriated magnetic field parameters during the laserMIG parameters $(2 \mathrm{~kW}, 160 \mathrm{~A}, 2 \mathrm{~m} / \mathrm{min})$ is concluded as $\mathrm{B}=24 \mathrm{mT}$.

Keywords: Transverse magnetic field $\cdot$ Laser-MIG hybrid welding-Ti-6Al-4V·Droplet transfer·Arc characteristics

\section{Introduction}

Titanium alloys have been used in the aerospace, electronic communication, precision instrument, petrochemical industry due to its low density, high specific strength, good ductility, and excellent high-temperature mechanical properties[1, 2]. Ti-6Al-4V is one of the most widely used titanium alloys due to its stable structure and good connection performance[3, 4]. Laser-arc welding has been recognized due to its excellent combination of welding advantages of both laser and arc such as good joint bridging performance, deep weldability, and high welding efficiency, et al.. The laser-arc hybrid welding is tried to weld Ti-6Al-4V in recent years.

However, there is still a dearth of studies on several problems in laser-MIG hybrid welding of Ti-6Al-4V, such as weld bead formation, welding stability and weld defects[7]. The studies in recent years indicated that the stability of the welding process and the welding bead appearance is influenced by arc performance[8] and droplet transfer[9] 
behavior. Liu et al.[10] indicates that the distance of the laser and the arc (DLA) could affect the plasma behavior and the transfer mode, therefore, the welding penetration depth is changed. Zhang et al.[11] observes the plasma characteristic and droplet transfer in $\mathrm{CO}_{2}$ laser-GMAW-P hybrid welding. The results show that the changed electromagnetic force increased the droplet size and the droplet formation time, and eventually makes an offaxis droplet phenomenon. Wang et al.[12] shows that a curved channel is formed between the welding wire and the keyhole in the fiber laser-MIG hybrid welding for ZL114. Chen et al.[13] has investigated the influence of welding position in $\mathrm{CO}_{2}$ laser-arc hybrid welding. The result shows that the mean transfer frequencies affected the welding bead appearance. Tang[14] et al. Indicates that shielding gas increase can make the plasma temperature increase. As a result, the penetration depth and weld width increases in the laser-MIG hybrid welding. Considering the above studies, it is confirmed that the arc performance and metal transfer behavior has a significant impact on the microstructure properties, welding penetration depth, welding bead appearance.

The arc performance and droplet transfer behavior must be managed to control the weld bead quality. The magnetic field have been used to regulate and control the welding process especially through controlling the arc performance and droplet behavior. In laser welding, the welding process will be affected when the magnetic field threshold exceeds $200 \mathrm{mT}$. Chen et al.[17] has investigated the influence of $240 \mathrm{mT}$ magnetic field for the laser welding of dissimilar materials. It is reported that the $240 \mathrm{mT}$ magnetic field is a benefit for the weld bead appearance and weld microstructure for the welding of the dissimilar materials. Avilov et al.[18] observes the welding bead appearance under different alternating magnetic fields in the laser beam welding. It concludes that the alternating magnetic field can produce a beneficial effect to inhibit gravitational drop-out 
and sagging of the weld. However, the welding process will be considerably affected when the magnetic field threshold is less than $50 \mathrm{mT}$ for arc welding. Guan et al.[19] indicates that external magnetic field (12.5 $\mathrm{mT}-17 \mathrm{mT})$ frequency had a significant impact on the arc shape and arc movement, which has a considerable influence on arc temperature gradient and under-cooling degree, which finally affected the weld appearance and mechanical properties in GMAW welding. Chang et al.[20] indicated that the synchronous electromagnetic field, including low-frequency magnetic fields for the arc-burning phase and high-frequency magnetic fields for the short-circuit phase can be introduced to the short-circuit GMAW to improve the frequency of metal transfer and reduce the spatter. Sun et al.[21] reported that the magnetic arc oscillation could result in the change of arc voltage and make the weld current flowing through sidewalls, which in turn caused the increase of sidewall penetration and redistribution of arc heat. Wang et al.[22] finds arc pressure of the bottom decreases while that of the sidewall via transverse magnetic field (4 mT) improved morphology through TIG welding. The voltage of the magnetic arc is invariably higher than that of non-magnetic arc, and the difference decreases with the increasing welding current. Moreover, the laser-MIG hybrid welding is also profoundly influenced by the external magnetic field when the magnetic field threshold is less than 50mT. Zhang et al.[23] shows that the external longitudinal magnetic field $(16 \mathrm{mT})$ can increase the the stability of welding arc and affect the force on droplet transfer in the laser-MIG hybrid welding of 316L. Zhu et al.[24] indicates that the combined effect of laser and magnetic field (16 mT) induces a periodically altered the axial asymmetry of arc along the welding direction in the laser-MIG hybrid welding of 316L. It is confirmed that the appropriate magnetic field has a considerable impact on the arc performance and droplet behavior in the laser-MIG hybrid welding through the literature above. 
However, there are few systematic studies on arc performance and metal transfer in transverse magnetic field-assisted Laser-MIG hybrid welding of Ti-6Al-4V Titanium alloy. This work studies the influence of the external magnetic field on the arc performance and droplet transfer in laser-MIG hybrid welding for Ti-6Al-4V, which can provide further guidance to improve the welding stability and weld quality.

\section{Experimental procedures}

\subsection{The materials}

The experimental materials are the Ti-6Al-4V Titanium alloy base metal with the thickness of $4 \mathrm{~mm}$ and the Ti-6Al-4V filler wire of $1 \mathrm{~mm}$ in diameter. The chemical compositions of the base metal and the wire are shown in Table 1. The acetone is used to remove the oil contamination on the surface of the sheets before welding. And then the surface treatment is carried out by laser cleaning with the $300 \mathrm{~W}$ laser devices to remove oxidation film and residual acetone. In order to protect the welding joint from oxidation, the pure argon with the flow rate of $2 \mathrm{~m}^{3} / \mathrm{h}$ is provided as the shielding atmosphere.

\subsection{Welding preparation}

A 4 kW fiber laser (IPG RYL-4000), which can provide a continuous wave output with $1.07 \mu \mathrm{m}$ laser emission wavelength is used to weld Ti-6Al-4V plates by bead welding. The laser spot with a $0.3 \mathrm{~mm}$ diameter is focused in the center of the sheet. A Fronius TPS 4000 digital power is used as the MIG welding power controller. The transverse external magnetic field is provided by the permanent magnet blocks, which can provide the maximum magnetic field of $60 \mathrm{mT}$. To ensure the stability of the external magnetic field and convenient for High-speed camera (HSC) shoot, the sheets are put between two magnet blocks as shown in Fig. 1. The magnetic block and the laser head keep stationary 
during welding. Therefore, the distribution of the magnetic induction lines is supposed to be perpendicular to the welding direction. The magnetic field intensity is adjusted by changing the distance between the magnetic block and weld plate. During the welding process, the control variable method is used to study the effect of magnetic field on droplet transfer and arc performance. The main welding parameters during hybrid welding is shown in Table 2. The HSC is placed in the position perpendicular to the welding direction and the lens parallels to the welding joint to observe the droplet and the arc.

\subsection{Image processing}

At first, the images got from HSC are handled through segmentation to reduce the influence of the laser plasma and the molten pool. Then, graying processing and media filtering is used to eliminate the influence of the surrounding laser plasma and other noises. Next, binarization is used to get the area of the arc. At last, the statistic result of pixels number is output to get the area of the arc. The frequency of HSC is $5000 \mathrm{~Hz}$, where each image stands for $0.2 \mathrm{~ms}$. The detailed information is shown in Fig. 2.

The number of pixels within $1 \mathrm{~mm}^{2}$ is output to calculate the total arc area. Median filter, peak seeking, elimination of accompanying peaks and curve fitting are used to get the mean maximum area and the variance of arc area under different magnetic field.

\section{Theoretical background}

In the laser-MIG hybrid welding, the performance of the weld cross-section is mainly influenced by the heat resource, the weld pool flowing and the droplet transfer. The heat input $(Q)$ is mixed by the laser heat input ( $\left.Q_{\text {laser }}\right)$ and the arc heat input $\left(Q_{\text {arc }}\right) . Q$ can be expressed by the following equation[5]:

$Q=Q_{\text {laser }}+Q_{\text {arc }}=\frac{\eta_{1} P_{\text {laser }}+\eta_{2} U I}{v}$ 
where $P$ is the power of the laser, $U$ is the arc voltage, $I$ is the welding current, $\eta_{1}$ is the efficiency of the laser beam, $\eta_{2}$ is the efficiency of the arc power, $v$ is the welding speed.

Many forces act on the droplet during the hybrid welding, such as the surface tension, gravity, the plasma flow force, the electromagnetic force and the external magnetic force. The forces acting on the droplet in the magnetic assisted hybrid welding are shown in Fig. 3.

There is the surface tension force between the molten metal and the plasma with the melting of welding wire. The surface tension force can be described by the following equation:

$F_{\sigma}=2 \pi r_{w} \sigma$

where $r_{w}$ stands for the wire radius, $\sigma$ means the surface tension coefficient.

The gravity can be described by the following equation:

$F_{g}=\frac{4}{3} \pi r^{3} \rho g$

where the $r$ stands for the droplet radius, $\rho$ means the droplet density, $g$ is the acceleration of the gravity.

There is metal vapor during the welding process, the force caused by this process can be described by the following equation:

$F_{v}=\left\{\begin{array}{l}\frac{1}{4 \pi R_{h}^{2}} C_{D} A \rho_{m}^{2} V_{0}^{2}\left(\frac{N_{a} K_{B} T_{s}^{3 / 2}}{M_{a} B_{0}}\right) \exp \left(-U / T_{s}\right) \exp \left(-D^{2} / 2 R_{h}^{2}\right)\left(D \leq R_{h}\right) \\ O\left(D \geq R_{h}\right)\end{array}\right\}$

where the $R_{\mathrm{h}}$ is the distribution parameters of metal vapor, $V_{0}$ is a constant, $N_{\mathrm{a}}$ is the Avogadro's constant, $K_{\mathrm{B}}$ is the Boltzmann constant, $T_{\mathrm{s}}$ is the surface temperature of the 
molten zone, $M_{\mathrm{a}}$ is the molecular weight, $U$ is a calculated constant, and $D$ is the distance between laser and arc.

One of the most important forces that act on the droplet during the hybrid welding process is the electromagnetic force. This force is produced by the current passed through the droplet and the welding arc. It can be described by the following equation:

$$
F_{e m}=\frac{\mu_{0} I^{2}}{4 \pi}\left[\ln \frac{r \sin \theta}{r_{w}}-\frac{1}{4}-\frac{1}{1-\cos \theta}+\frac{2}{(1-\cos \theta)^{2}} \ln \frac{2}{1+\cos \theta}\right]
$$

where $I$ is the welding current, $\mu_{0}$ is the permeability of free space, $r$ and $r_{\mathrm{w}}$ stand for the radius of the droplet and the wire, $\theta$ is the angle of the arc-cover area.

The extra magnetic force is one of the most important force when the magnetic field act on the hybrid welding process. This force is produced by the external Lorentz force that the external magnetic field acting on the current passed through the droplet and the welding arc. This force can be described by the following equation:

$$
F_{B}=\frac{B I \int_{2 r}^{0} L d l}{r}=\frac{\pi B I r}{2}
$$

where $B$ stands for the extra magnetic field magnitude, $I$ is the welding current, $L$ is the length of arc influence area, $r$ is the radius of the droplet.

\section{Result and Discussion}

\subsection{Effects of external magnetic field on weld appearance}

The effects of different external magnetic field on weld appearance are shown in Table 3. The weld appearance without an external magnetic field under high-speed welding $(2 \mathrm{~m} / \mathrm{min})$ is not uniform and straight. There are obvious incomplete fusion 
regions and undercut, which are marked in the images. This welding appearance may be caused by insufficient droplet transfer frequency during high-speed welding. The weld quality under $8 \mathrm{mT}$ to $24 \mathrm{mT}$ indicates that an appropriate external magnetic field has a positive influence on the weld appearance. The figures show that the incomplete fusion gradually disappears, the weld becomes straight and uniform with the increase of magnetic field intensity. This phenomenon indicates the weld quality is improved under appropriate external magnetic field. The welding defects such as spatter reappears, the weld profile is not uniform under the excessive external magnetic field. This phenomenon shows that the excessive external magnetic field has a negative impact on the weld quality.

\subsection{Effects of external magnetic field on arc characteristics}

\subsubsection{Effects of external magnetic field on the performance of stable arc}

The effects of different external magnetic field on the performance of stable arc is shown in Fig. 4. During hybrid welding, laser is able to attract and stabilize the arc. The $d\left(d=d_{1}+d_{2}\right)$ is the arc length, which represents the arc shrinkage. And the $d_{1}$ and $d_{2}$ indicate the forward and backward arc length during the hybrid welding respectively. The external magnetic is benefit for arc shrinkage when $d_{1}>d_{2}$, else, the magnetic field promotes arc divergence and unstability. The figures indicate that the $d, d_{1}$, and $d_{2}$ are inconstant value under different magnetic field. Therefore, the average of $d$ must be calculated to identify the arc performance during the hybrid welding.

The arc is always towards the laser direction without the external magnetic field, as shown in Fig. 4a. $d$ is changed by $8.35 \%$ from $8.1 \mathrm{~mm}$ to $8.8 \mathrm{~mm}$ in four adjacent cycles, which means that the arc is unstable during this hybrid welding process. Meanwhile, $d_{1}>d_{2}$, the arc is in the forward situation by the attraction of the laser. Fig. $4 \mathrm{~b}-4 \mathrm{c}$ show the 
$8 \mathrm{mT}$ and $16 \mathrm{mT}$ external magnetic field are able to stabilize arc. The $d$ decreases from $5.3 \mathrm{~mm}$ to $4.3 \mathrm{~mm}$ with the increase of the magnetic field, meanwhile, $d_{1}>d_{2}$. The arc spreads perpendicular to the welding direction rather than towards the laser direction, which shrinks and condenses at the end of the welding wire with the increase of the magnetic field. At $24 \mathrm{mT}$ (Fig. $4 \mathrm{~d}$ ), $d$ is $2.7 \mathrm{~mm}$ and $d_{1}=d_{2}$, where the arc is compressed to the maximum extent. Arc shrinkage makes energy aggregation, increases unit energy density and energy utilization efficiency, and leads to uniform weld and penetration increasing. The shape of arc changes from bell to dish and the arc length is very short, which indicates the droplet transfer form changes from short-circuit transfer to mesospray transfer. Fig. 4e shows the influence of $32 \mathrm{mT}$ external magnetic fields on the performance of arc. $d$ is $4.1 \mathrm{~mm}$ and larger than it under $24 \mathrm{mT}$ magnetic field, meanwhile, $d_{1}<d_{2}$. It means that arc diffuses compared with the arc under $24 \mathrm{mT}$ magnetic field. Arc deflection is more serious in this set of experimental parameters.

In conclusion, the images show that the arc is unstable during the high-speed hybrid welding process, appropriate external magnetic field $(24 \mathrm{mT})$ is able to effectively improve arc stability and condenses arc. Conversely, excessive external magnetic (32 mT) field makes arc divergence and arc deflection becomes serious.

\subsubsection{Analysis of the influence of external magnetic field on the arc characteristics}

Arc is a strong and lasting discharge phenomenon in the gas medium between two electrodes or between electrodes and base metal with a certain voltage. The physical nature of arc is charged particles along with the current and regarded as the aggregation of electric streamline. Electric streamline can be deeply affected by external magnetic field. External Lorentz force $F_{B}$ and electromagnetic force $F_{\mathrm{em}}$ is the most important forces during hybrid welding. 
In laser-MIG hybrid welding process, the main force act on arc electric streamline is the electromagnetic force $F_{\mathrm{em}}$. It is from laser to droplet during this process. $F_{\mathrm{em}}$ is the force along electric streamline. Arc is attracted by laser and towards the welding forward direction. $F_{\text {em }}$ is totally from laser keyhole to droplet. The arc length is closed to $D L A$, as is shown in Fig. 5a. When the appropriate magnetic field is added, arc is affected by the external Lorentz force $F_{\mathrm{B}}$. According to the left-hand rule, this force is perpendicular to the electric streamline. External Lorentz force on forward electric streamline is backward and downward. Conversely, the external Lorentz force on backward electric streamline is backward and upward. This phenomenon makes the electric streamline in arc rotating away from the laser direction. Therefore, arc shrinks and gathers under the welding wire when the resultant force of external Lorentz force is backward and downward especially under $24 \mathrm{mT}$ external magnetic field. This process can improve the stability of arc and the energy efficiency, as shown in Fig. 5b. However, with the increase of the external magnetic field, arc gradually moves against the welding direction. When the excessive magnetic field $(32 \mathrm{mT})$ is added, the direction of the resultant external Lorentz force for arc is backward and upward. The heat on the wire increases because arc gathers at the end of the wire and climbs along the wire when the $F_{\mathrm{B}}$ is upward. The arc is separated into two parts. Finally, arc becomes unstable. The influence of the excessive magnetic field on the arc performance is shown in Fig. $5 \mathrm{c}$.

\subsubsection{Effects of magnetic field on the maximum of stable arc and arc stability}

The image processing is used to acquire the change of arc area with time in different external magnetic field, as shown in Fig. 6. The relationship between arc area and time without external magnetic field is shown in Fig. 6a. The cycle of arc area change is unstable value and the maximum area of the static arc is obvious gradient changed. The 
average arc change cycle is $88.31 \mathrm{~ms}$ and the maximum of arc area is $89.83 \mathrm{~mm}^{2}$, which indicates a long arc change cycle and arc diffusion in this phenomenon. The arc disappears for a long time at the end of each cycle, which stands for the long arc extinguishing time and arc unstability at this time. At $8 \mathrm{mT}$ in Fig. $6 \mathrm{~b}$, the cycle of the arc area change is $61.91 \mathrm{~ms}$ and the maximum area is $58.87 \mathrm{~mm}^{2}$, which indicates that amplitude becomes smaller and the cycle becomes shorter comparing with that under 0 $\mathrm{mT}$. However, long arc extinguishing time is exiting during the welding process under 8 mT external magnetic field. The amplitude becomes smaller and the long arc extinguishing time disappears when the amplitude of the external magnetic field reaches $16 \mathrm{mT}$ and $24 \mathrm{mT}$ in Fig. 6c-6d. The cycle of arc area has a little change from $46.33 \mathrm{~ms}$ to $44.61 \mathrm{~ms}$, which means that the change of arc area during this process become more uniform. Fig. 6e at $32 \mathrm{mT}$ indicates that long arc extinguishing time reappears in each cycle and the area of arc mutation occurs. The maximum area of arc is $175.81 \mathrm{~mm}^{2}$, which is much bigger than that under $0 \mathrm{mT}$. The stability of arc becomes weak under these parameters. Besides, the surface quality of welding beam is affected.

The variance of arc area average peak value stands for the stability of arc and the average peak value of arc area stands for the property of arc shrinkage. As is shown in Fig. 7, the average peak value of arc size goes down by $58.5 \%$ from $37.33 \mathrm{~mm}^{2}$ to 15.49 $\mathrm{mm}^{2}$ with the magnetic field increasing from $0 \mathrm{mT}$ to $16 \mathrm{mT}$. And it goes up by $54.8 \%$ from $15.49 \mathrm{~mm}^{2}$ to $23.99 \mathrm{~mm}^{2}$ with the magnetic field increasing from $16 \mathrm{mT}$ to $32 \mathrm{mT}$. In summary, it means that arc shrinks with the addition of the magnetic field and diffuses under the excessive magnetic field. The variance of arc area average peak value goes down by $62.3 \%$ from $2.81 \mathrm{~mm}^{2}$ to $1.06 \mathrm{~mm}^{2}$ with the magnetic field changing from $0 \mathrm{mT}$ to $24 \mathrm{mT}$ and it goes up by $74.5 \%$ from $1.06 \mathrm{~mm}^{2}$ to $1.85 \mathrm{~mm}^{2}$ with the magnetic field 
changing from $24 \mathrm{mT}$ to $32 \mathrm{mT}$. This phenomenon indicates that arc stability improves with the addition of appropriate magnetic field $(8-24 \mathrm{mT})$ and decreases under the excessive magnetic field (32 $\mathrm{mT})$.

In conclusion, with the increasing of appropriate external magnetic field (8-24 mT), the cycle of arc area change becomes shorter, the amplitude becomes smaller and the change of arc area tends to be more uniform. The maximum area of arc is smaller than it without external magnetic, which indicates arc shrinks under the action of external magnetic field. Conversely, the arc stability becomes worse under the excessive external magnetic field $(32 \mathrm{mT})$. The maximum area of stable arc becomes larger than that under the appropriate magnetic field. The appropriate magnetic field parameters is $24 \mathrm{mT}$.

\subsection{Effects of external magnetic field on droplet transfer behavior}

\subsubsection{Effects of external magnetic field on droplet transfer performance}

The droplet transfer process is shown in Fig. 8. At first, the droplet is towards laser. Droplet grows up and the direction is consistent with welding wire direction with time. This droplet transfer form is short-circuit transfer. The droplet grows slowly on the welding wire and separates from the wire when it is contacting with the molten pool. The wire is directly contacting with the molten pool. This transfer form tends to produce spatter and hump.

The droplet transfer performance under different external magnetic fields is shown in Fig. 9. The droplet behavior under high-speed hybrid welding without an external magnetic field is shown in Fig. 9a. The diameter of droplet is $1.835 \mathrm{~mm}$, which indicates that the droplet detachment size is large. In Fig. $9 \mathrm{~b}$ at $8 \mathrm{mT}$, the external magnetic field is not enough to change the droplet transform, the droplet is towards weld plate. The droplet 
detachment diameter is $1.578 \mathrm{~mm}$, which is bigger than the wire diameter. The droplet transfer performance at $16 \mathrm{mT}$ is similar to the performance at $8 \mathrm{mT}$, as shown in Fig. 9c. The external magnetic field tends to effectively improve droplet transfer efficiency when the amplitude of external magnetic field reaches $24 \mathrm{mT}$ in Fig. 9d. The droplet is inclined downward and backward. The droplet detachment size is $1.033 \mathrm{~mm}$, which is closed to that of wire. The form of the droplet transfer changes from short-current transfer to mesospray transfer and the frequency of droplet transfer increases. Fig. 9e shows the influence of $32 \mathrm{mT}$ external magnetic fields on droplet. The droplet detachment diameter is 1.098 $\mathrm{mm}$ and the frequency becomes higher. However, the stability of droplet transfer becomes worse in this process. The droplet is divided into two parts and one part moves upward along the welding wire. The shape of detachment droplet is unstable which tends to produce incomplete fusion welding defect.

It can be identified from Fig. 9 that the droplet size under $0 \mathrm{mT}$ is larger than it under $8 \mathrm{mT}-24 \mathrm{mT}$, and the droplet transfer at $32 \mathrm{mT}$ is unstable. It means that the droplet spreads unevenly on weld beam under high welding speed. The experiment can be divided into three cases:case 1, insufficient droplet transfer; case 2, appropriate droplet transfer; case 3, excessive droplet transfer. Welding quality is insufficient in case 1 or case 3 and excellent in case 2 . However, the mechanism in case 1 and case 3 is different. The reason for case 1 is that surface tension force is too large that droplet is not able to detach from the wire, and the reason for case 3 is that the wire melting speed is too fast to fit the droplet transfer process.

In conclusion, the trajectory of droplet changes with increase of external magnetic field (case 2). The suitable external magnetic field ( $24 \mathrm{mT})$ is able to efficiently improve the droplet transfer frequency and decrease the droplet detachment size. The small 
magnetic field $(8 \mathrm{mT})$ is not available to change the droplet transfer form and the excessive external magnetic field (case 3) decreases droplet transfer process stability. The appropriate magnetic field parameters is $24 \mathrm{mT}$.

\subsubsection{Analysis for the influence of magnetic field on droplet transfer behavior}

The main force acting on the droplet during the droplet transfer process can be identified through the theoretical background. However, the droplet behavior and the forces acting on the droplet will change with the increase of the external magnetic field. The direction and the magnitude of these forces should be identified clearly to study the relationship between droplet behavior and the external magnetic field.

The direction of the forces depends on the location of the welding head. The forces on the droplet during the MIG welding are shown in Fig. 10a. When the laser is added, the forces on the droplet are shown in Fig. 10b. When the external magnetic field is added during the hybrid welding, the forces acted on the droplet are displayed as Fig. 10c. The direction of $F_{g}$ is vertically downward and magnitude of it increases with the droplet growing up. $F_{g}$ reaches its maximum value before the droplet detachment and it can be calculated by the formula (3). The direction of $F_{v}$ is consistent with the steam movement direction and it is vertically upward. According to formula (4), $F_{v}$ is negligible when the $D L A$ is larger than the diameter of the droplet. The direction of $F_{e m}$ is determined by the arc characteristics and the position of the droplet, whose direction is parallel to the arc direction. The value of $F_{e m}$ can be calculated by the formula (5). The $F_{\sigma}$ acts on the interfaces among solid, liquid and gas. Its direction points to the contraction direction at the tangent of the interfaces. There are three interface types (liquid-gas, solid-liquid, gassolid) and the direction of the resultant force among the whole interfaces is from the center of the droplet to the welding wire. It can be calculated by the formula (2). The external 
magnetic field is a transverse magnetic field. According to the left-hand rule, the direction of $F_{\mathrm{B}}$ is perpendicular to the current direction, backward and downward. $F_{B}$ is calculated by the formula (6).

As is shown in Fig. 11, the maximum of the droplet detachment size, the angle of the arc-cover area and the detachment cycle are influenced by the external magnetic field. These information from the HSC images are used to calculate the forces on the droplet under different magnetic fields. The values of forces under different external magnetic field are shown in Table 4.

The droplet detachment criterion is described by the equation below:

$\vec{F}_{\mathrm{D}}=\vec{F}_{g}+\vec{F}_{e m}+\vec{F}_{v}+\vec{F}_{B}>\vec{F}_{\sigma}$

The droplet transfer form will be the short-circuit transfer if $F_{D}<F_{\sigma}$ before the droplet contacts the molten pool. In this situation, the surface tension $F_{\sigma m}$ between droplet and molten pool promotes the droplet detachment. This force is determined by the contact area between droplet and molten pool. The contact area between droplet and molten pool is proportional to the contact time. Therefore, the contact time between droplet and molten pool is proportional to the difference between $F_{D}$ and $F_{\sigma}$.

The forces acted on the droplet without the magnetic field are shown in Fig. 11a. $F_{\sigma}$ is larger than the resultant force of other forces $F_{D}$, the droplet can not be detached from the wire. The contact time between droplet and molten pool is so long that the stability of the droplet transfer decreases. The external Lorentz force $F_{B}$ is the detachment force and its direction is contrast to the $F_{\sigma}$. It can reduce the contact time between droplet and molten pool. When $B=8 \mathrm{mT}$, the forces acted on the droplet are shown in Fig. $11 \mathrm{~b}$. The $F_{B}$ is smaller than the $F_{\sigma}$, and the direction of the $F_{B}$ is almost perpendicular to the $F_{\sigma} . F_{B}$ 
has no significant impact on the droplet. With the increase of the magnetic field, the $F_{B}$ increases rapidly. When $B=16 \mathrm{mT}$, the forces acted on the droplet are similar to that at 8 $\mathrm{mT}$. The $F_{\mathrm{B}}$ is also smaller than the $F_{\sigma}$, and the difference is more than $50 \%$ of $F_{\sigma}$. When $B=24 \mathrm{mT}$, as shown in Fig. $11 \mathrm{c}$, the $F_{\sigma}$ is larger than the $F_{D}$ and the difference is $28.2 \%$ of $F_{\sigma}$. It promotes the best contact time between droplet and molten pool. The contact time is so small that droplet transfer mode changes from short circuit transfer to mesospray transfer. Therefore, the droplet transfer stability is improved. This phenomenon is mainly attributed to the change of Lorentz force direction during droplet rotation, which resultantly increases effective detachment energy. When $B=32 \mathrm{mT}$, the forces acted on the droplet are shown in Fig. 11d. The direction of $F_{\mathrm{B}}$ is backward and upward. This process is not conducive to droplet separation from wire. The droplet moves backward and divides into two parts. This magnetic field is excessive magnetic field. The droplet will move upward along the wire, which leads to the droplet department occurring. External magnetic field at $24 \mathrm{mT}$ can promote the effective detachment energy improvement and the droplet transfer stability.

\section{Conclusion}

The effect of the transverse magnetic field on the arc performance, droplet transfer and the weld bead appearance in the laser-MIG hybrid welding of Ti-6Al-4V are investigated in this work, especially, transverse magnetic fields with $0 \mathrm{mT}, 8 \mathrm{mT}, 16 \mathrm{mT}$, $24 \mathrm{mT}$ and $32 \mathrm{mT}$ are studied. The appropriated magnetic field parameters during the laser-MIG parameters $(2 \mathrm{~kW}, 160 \mathrm{~A}, 2 \mathrm{~m} / \mathrm{min})$ is concluded as $\mathrm{B}=24 \mathrm{mT}$. The main results are summarized as follows:

(1) The appropriate external magnetic field (case 2) will improve the weld quality and reduce the welding defects such as incomplete fusion and undercut. While, the 
excessive external magnetic field (case 3) leads to new welding defects such as spatter, hump and incomplete fusion.

(2) The arc performance depends on the distribution of the electric streamline. An appropriate transverse magnetic field $(24 \mathrm{mT})$ can shrink the arc and improve its stability. The shape of the arc changes from bell to dish. Arc shrinkage and stability affect the welding process stability and the wire melting speed.

(3) Droplet transfer cycle transfer size decreases, which leads to the reduction of the contact time between droplet and molten pool. Droplet transfer form changes from shortcircuit transfer to meso-spray transfer, which implies the droplet transfer stability increasing at $24 \mathrm{mT}$.

(4)The external magnetic field is mainly attributed to the change of Lorentz force direction during droplet rotation, which resultantly increases effective detachment energy, which leads to the reduction of the contact time between droplet and molten pool. Finally, it promotes the effective detachment energy improvement and the droplet transfer stability.

\section{Acknowledgments}

This research has been supported by National Natural Science Foundation of China (NSFC) under Grant No. 51861165202 and No. 51721092. The authors would also like to thank the anonymous referees for their valuable comments.

\section{Declarations}

Ethics approval Not applicable.

Consent to participate Not applicable.

Consent for publication Not applicable. 
Conflict of interest The authors declare that they have no conflict of interest.

\section{References}

[1] I. Gurrappa (2003) Characterization of titanium alloy Ti-6Al-4V for chemical, marine and industrial applications. Materials Characterization 51: 131-139. https://doi.org/10.1016/j.matchar.2003.10.006.

[2] P. Traverso, and E. Canepa (2014) A review of studies on corrosion of metals and alloys in deep-sea environment. Ocean Engineering 87: 10-15. https://doi.org/10.1016/j.oceaneng.2014.05.003.

[3] B. Chang, Z. Yuan, H. Pu, H. Li, H. Cheng, D. Du, and J. Shan (2017) A Comparative Study on the Laser Welding of Ti6Al4V Alloy Sheets in Flat and Horizontal Positions. Applied Sciences 7: https://doi.org/10.3390/app7040376.

[4] F. Caiazzo, F. Cardaropoli, V. Alfieri, V. Sergi, P. Argenio, and G. Barbieri (2017) Disk-laser Welding of Ti-6Al-4V Titanium Alloy Plates in T-joint Configuration. Procedia Engineering 183: 219-226.

https://doi.org/10.1016/j.proeng.2017.04.024.

[5] Z. Gao, P. Jiang, C. Wang, X. Shao, S. Pang, Q. Zhou, X. Li, and Y. Wang (2016) Study on droplet transfer and weld quality in laser-MIG hybrid welding of 316L stainless steel. The International Journal of Advanced Manufacturing Technology 88: 483-493. https://doi.org/10.1007/s00170-016-8774-2.

[6] X. Su, W. Tao, Y. Chen, and J. Fu (2018) Microstructure and Tensile Property of the Joint of Laser-MIG Hybrid Welded Thick-Section TC4 Alloy. Metals 8: https://doi.org/10.3390/met8121002.

[7] Y. Sun, G. Luo, J. Zhang, C. Wu, J. Li, Q. Shen, and L. Zhang (2018) Phase transition, microstructure and mechanical properties of TC4 titanium alloy prepared by plasma activated sintering. Journal of Alloys and Compounds 741: 918-926. https://doi.org/10.1016/j.jallcom.2018.01.197.

[8] W. Liwei, S. Yingchao, L. Zhimin, W. Dianlong, X. Jun, and Y. Dejun (2019) Numerical simulation and experiments on the behavior and mechanism of laserassisted droplet transfer. Optics and Lasers in Engineering 122: 303-311. https://doi.org/10.1016/j.optlaseng.2019.06.019.

[9] S. Liu, F. Zhang, S. Dong, H. Zhang, and F. Liu (2018) Characteristics analysis of droplet transfer in laser-MAG hybrid welding process. International Journal of Heat and Mass Transfer 121: 805-811. https://doi.org/10.1016/j.ijheatmasstransfer.2018.01.047. 
[10] L. M. Liu, S. T. Yuan, and C. B. Li (2013) Effect of relative location of laser beam and TIG arc in different hybrid welding modes. Science and Technology of Welding and Joining 17: 441-446. https://doi.org/10.1179/1362171812y.0000000033.

[11] W. Zhang, X. Hua, W. Liao, F. Li, and M. Wang (2014) Behavior of the plasma characteristic and droplet transfer in CO2 laser-GMAW-P hybrid welding. The International Journal of Advanced Manufacturing Technology 72: 935-942. https://doi.org/10.1007/s00170-014-5731-9.

[12] J. Wang, C. Wang, X. Meng, X. Hu, Y. Yu, and S. Yu (2011) Interaction between laser-induced plasma/vapor and arc plasma during fiber laser-MIG hybrid welding. Journal of Mechanical Science and Technology 25: 1529-1533. https://doi.org/10.1007/s12206-011-0410-3.

[13] Y. B. Chen, J. C. Feng, L. Q. Li, Y. Li, and S. Chang (2013) Effects of welding positions on droplet transfer in $\mathrm{CO} 2$ laser-MAG hybrid welding. The International Journal of Advanced Manufacturing Technology 68: 1351-1359. https://doi.org/10.1007/s00170-013-4926-9.

[14] G. Tang, H. Chen, X. Yang, and L. Shen (2018) Effects of different welding process on the electronic temperature of plasma and weld shape during laserMIG hybrid welding of A7N01P-T4 aluminum alloy. Journal of Laser Applications 30: https://doi.org/10.2351/1.5004434.

[15] J. Chen, Q. Chen, and C. Wu (2020) Study of high-speed pulsed gas metal arc welding assisted by external magnetic-field. Science and Technology of Welding and Joining 25: 564-570. https://doi.org/10.1080/13621718.2020.1774994.

[16] Z. Zhengwu, M. Xiuquan, W. Chunming, and M. Gaoyang (2020) Grain refinement and orientation alternation of $10 \mathrm{~mm} 316 \mathrm{~L}$ welds prepared by magnetic field assisted narrow gap laser-MIG hybrid welding. Materials Characterization 164: https://doi.org/10.1016/j.matchar.2020.110311.

[17] R. Chen, C. Wang, P. Jiang, X. Shao, Z. Zhao, Z. Gao, and C. Yue (2016) Effect of axial magnetic field in the laser beam welding of stainless steel to aluminum alloy. Materials \& Design 109: 146-152.

https://doi.org/10.1016/j.matdes.2016.07.064.

[18] V. Avilov, A. Fritzsche, M. Bachmann, A. Gumenyuk, and M. Rethmeier (2016) Full penetration laser beam welding of thick duplex steel plates with electromagnetic weld pool support. Journal of Laser Applications 28: https://doi.org/10.2351/1.4944103.

[19] Z. Q. Guan, H. X. Zhang, X. G. Liu, A. Babkin, and Y. L. Chang (2019) Effect of magnetic field frequency on the shape of GMAW welding arc and weld 
microstructure properties. Materials Research Express 6:

https://doi.org/10.1088/2053-1591/ab2572.

[20] Y. L. Chang, X. L. Liu, L. Lu, A. S. Babkin, B. Y. Lee, and F. Gao (2013) Impacts of external longitudinal magnetic field on arc plasma and droplet during short-circuit GMAW. The International Journal of Advanced Manufacturing Technology 70: 1543-1553. https://doi.org/10.1007/s00170-013-5403-1.

[21] Q. Sun, J. Wang, C. Cai, Q. Li, and J. Feng (2015) Optimization of magnetic arc oscillation system by using double magnetic pole to TIG narrow gap welding. The International Journal of Advanced Manufacturing Technology 86: 761-767. https://doi.org/10.1007/s00170-015-8214-8.

[22] J. Wang, Q. Sun, J. Feng, S. Wang, and H. Zhao (2016) Characteristics of welding and arc pressure in TIG narrow gap welding using novel magnetic arc oscillation. The International Journal of Advanced Manufacturing Technology 90: 413-420. https://doi.org/10.1007/s00170-016-9407-5.

[23] X. Zhang, Z. Zhao, G. Mi, C. Wang, R. Li, and X. Hu (2017) Effect of external longitudinal magnetic field on arc plasma characteristics and droplet transfer during laser-MIG hybrid welding. The International Journal of Advanced Manufacturing Technology 92: 2185-2195. https://doi.org/10.1007/s00170-0170293-2.

[24] Z. Zhu, X. Ma, C. Wang, and G. Mi (2020) Modification of droplet morphology and arc oscillation by magnetic field in laser-MIG hybrid welding. Optics and Lasers in Engineering 131: https://doi.org/10.1016/j.optlaseng.2020.106138. 

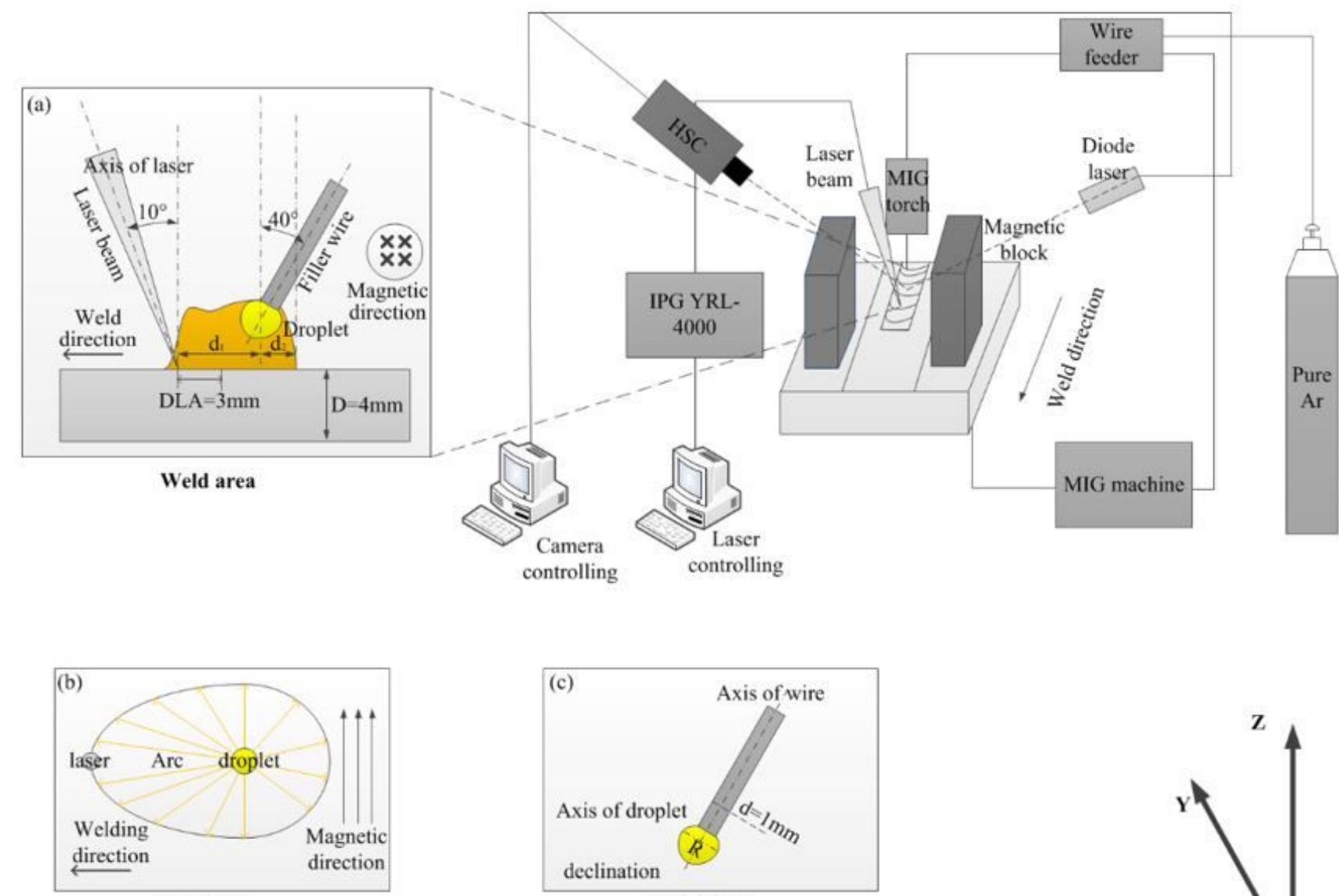

Top View

MIG Torch

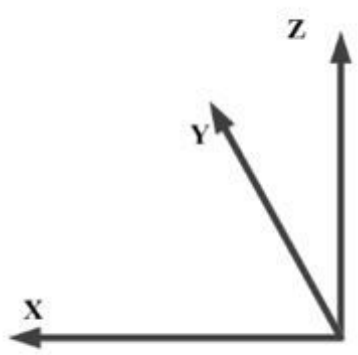

Figure 1

Schematic of the magnetic field assisted laser-MIG hybrid welding (a) the magnetic field assisted laserMIG welding system (b) the top view of the weld structure (c) the weld torch structure 


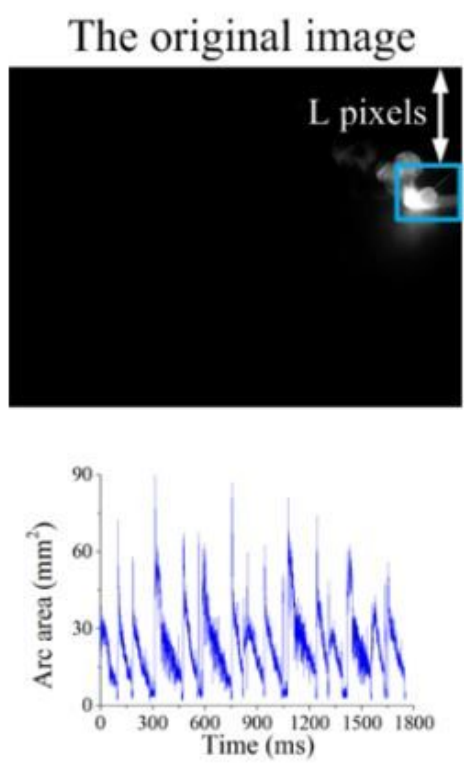

Arc area

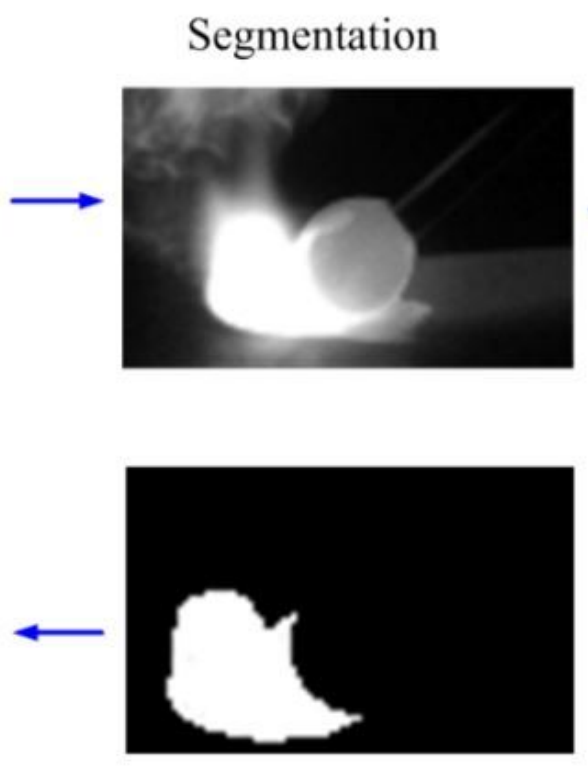

Binarization
Graysacle processing

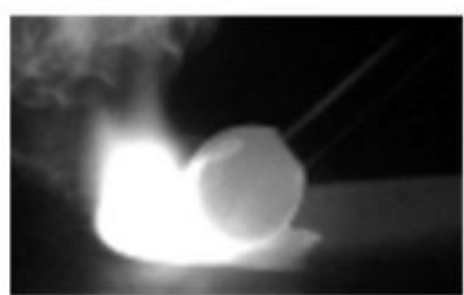

1

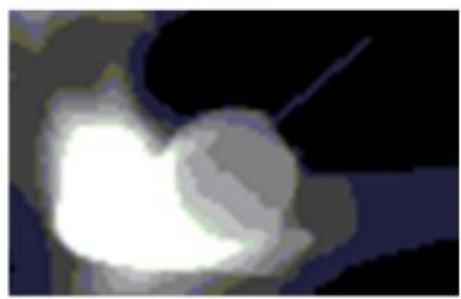

Median filtering

Figure 2

The arc features extraction processing

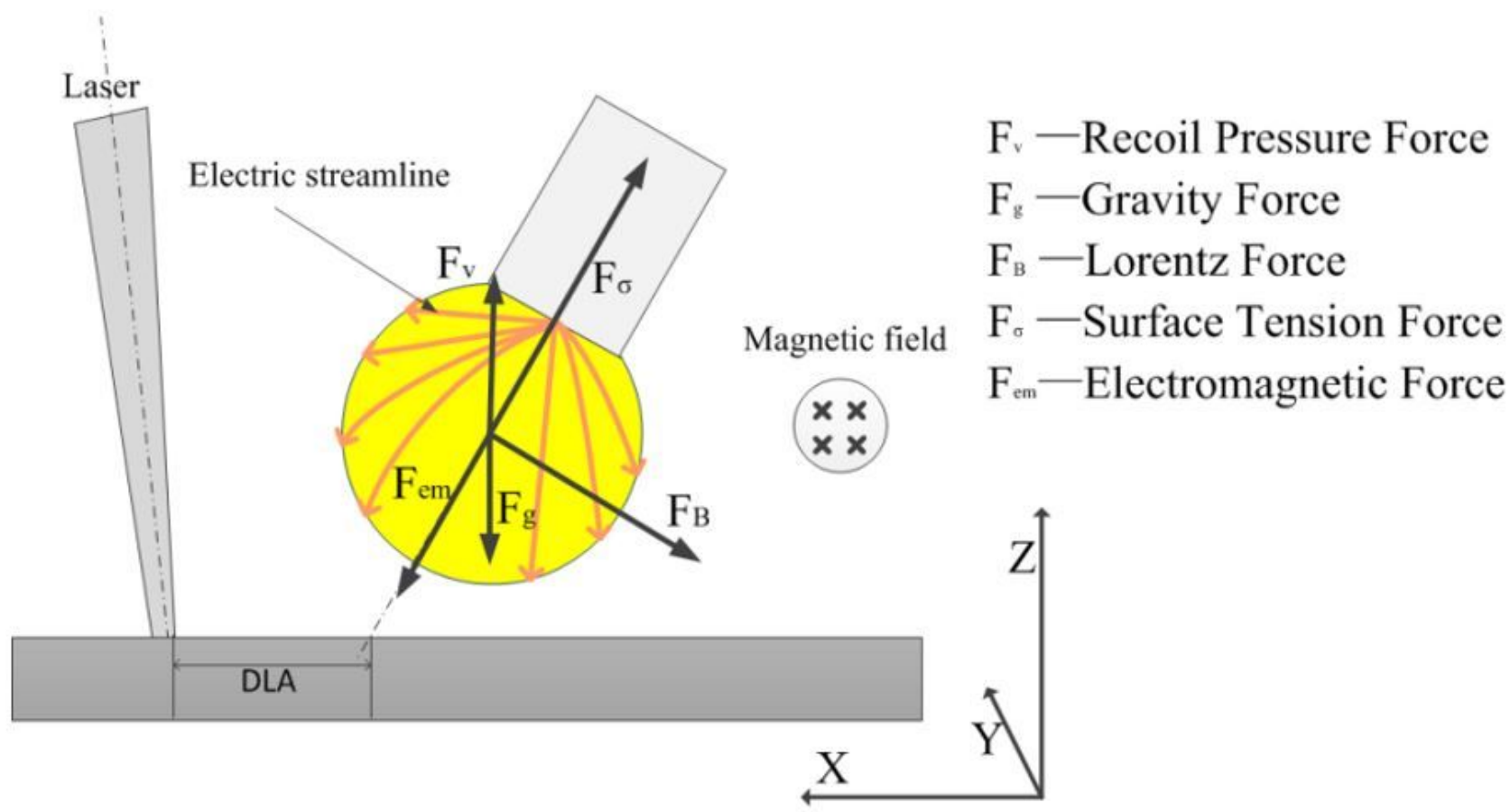

Figure 3

Forces acting on the droplet in the transverse magnetic field-assisted Laser-MIG hybrid welding 


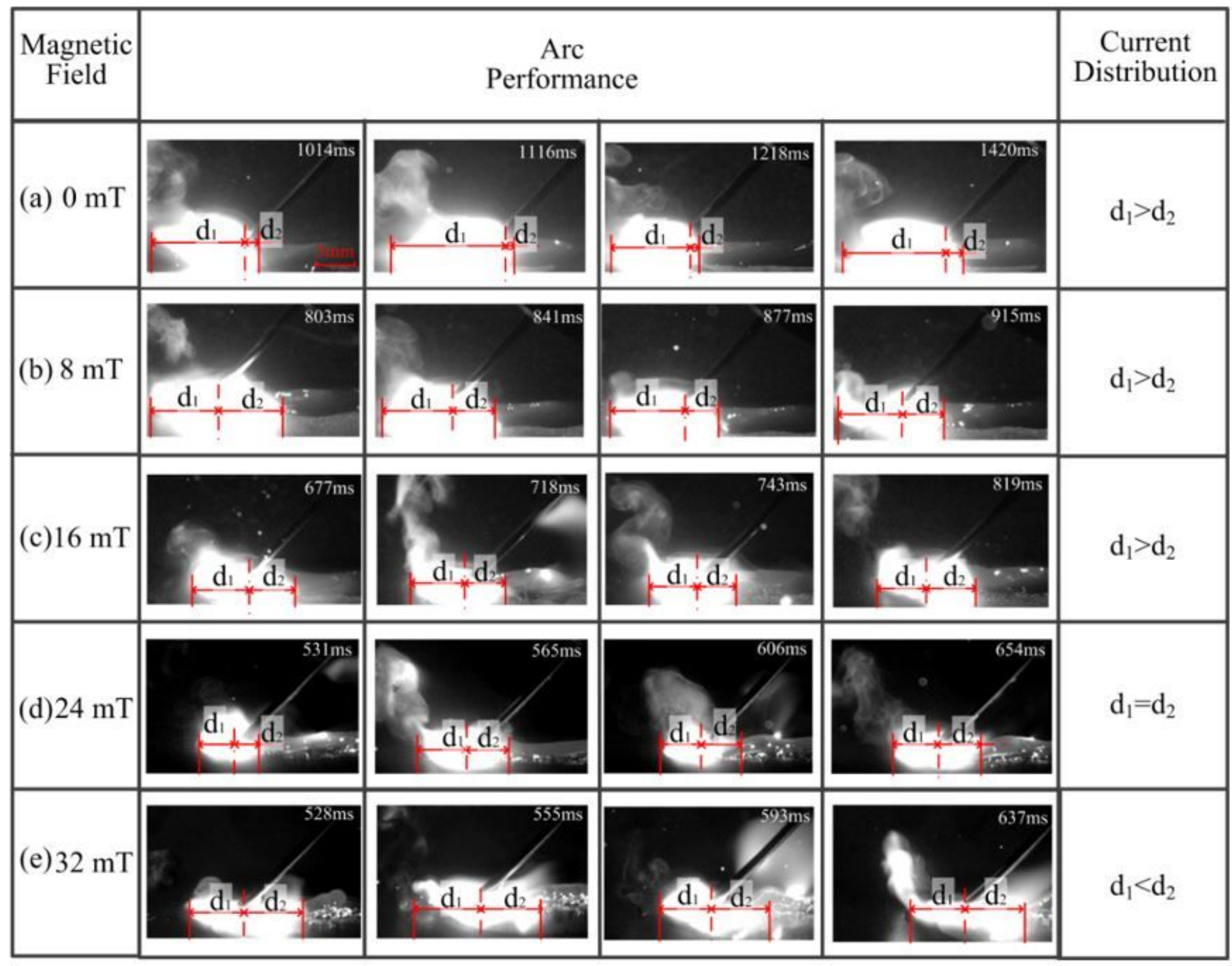

Figure 4

Effects of external magnetic field on the performance of the arc 


\begin{tabular}{|c|c|c|c|}
\hline & (a) $\begin{array}{c}\text { Without } \\
\text { Magnetic field }\end{array}$ & (b) $\begin{array}{c}\text { Appropriate } \\
\text { Magnetic field }\end{array}$ & (c) $\begin{array}{c}\text { Excessive } \\
\text { Magnetic field }\end{array}$ \\
\hline $\begin{array}{c}\text { Electric } \\
\text { streamline }\end{array}$
\end{tabular}

\section{Figure 5}

The external Lorentz force on the arc under a different magnetic field, the arc performance changed with the different magnetic field with the Lorentz force. 


\begin{tabular}{|c|c|c|c|}
\hline $\begin{array}{c}\text { Magnetic } \\
\text { flied }\end{array}$ & & $\begin{array}{c}\text { Arc area vs time } \\
\text { diagram }\end{array}$ & $\begin{array}{l}\text { Arc change } \\
\text { period }\end{array}$ \\
\hline $0 \mathrm{mT}$ & (a) & Time (ms) & $88.31 \mathrm{~ms}$ \\
\hline $8 \mathrm{mT}$ & (b) & 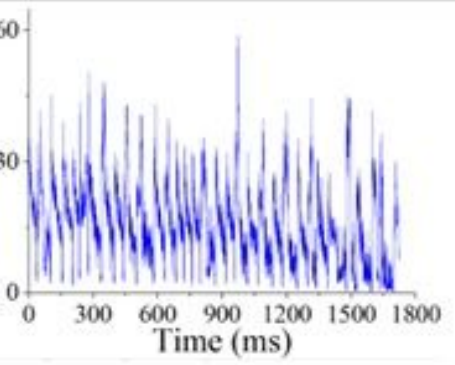 & $61.19 \mathrm{~ms}$ \\
\hline $16 \mathrm{mT}$ & (c) & $\frac{{ }_{0}^{90}}{300-\frac{600}{900} 1200 \quad 1500 \quad 1800}$ & $46.33 \mathrm{~ms}$ \\
\hline $24 \mathrm{mT}$ & (d) & $\frac{{ }_{0}^{20}}{30-1}$ & $44.61 \mathrm{~ms}$ \\
\hline $32 \mathrm{mT}$ & (e) & Time (ms) & $27.38 \mathrm{~ms}$ \\
\hline
\end{tabular}

Figure 6

Effects of external magnetic field on the change of the arc area 


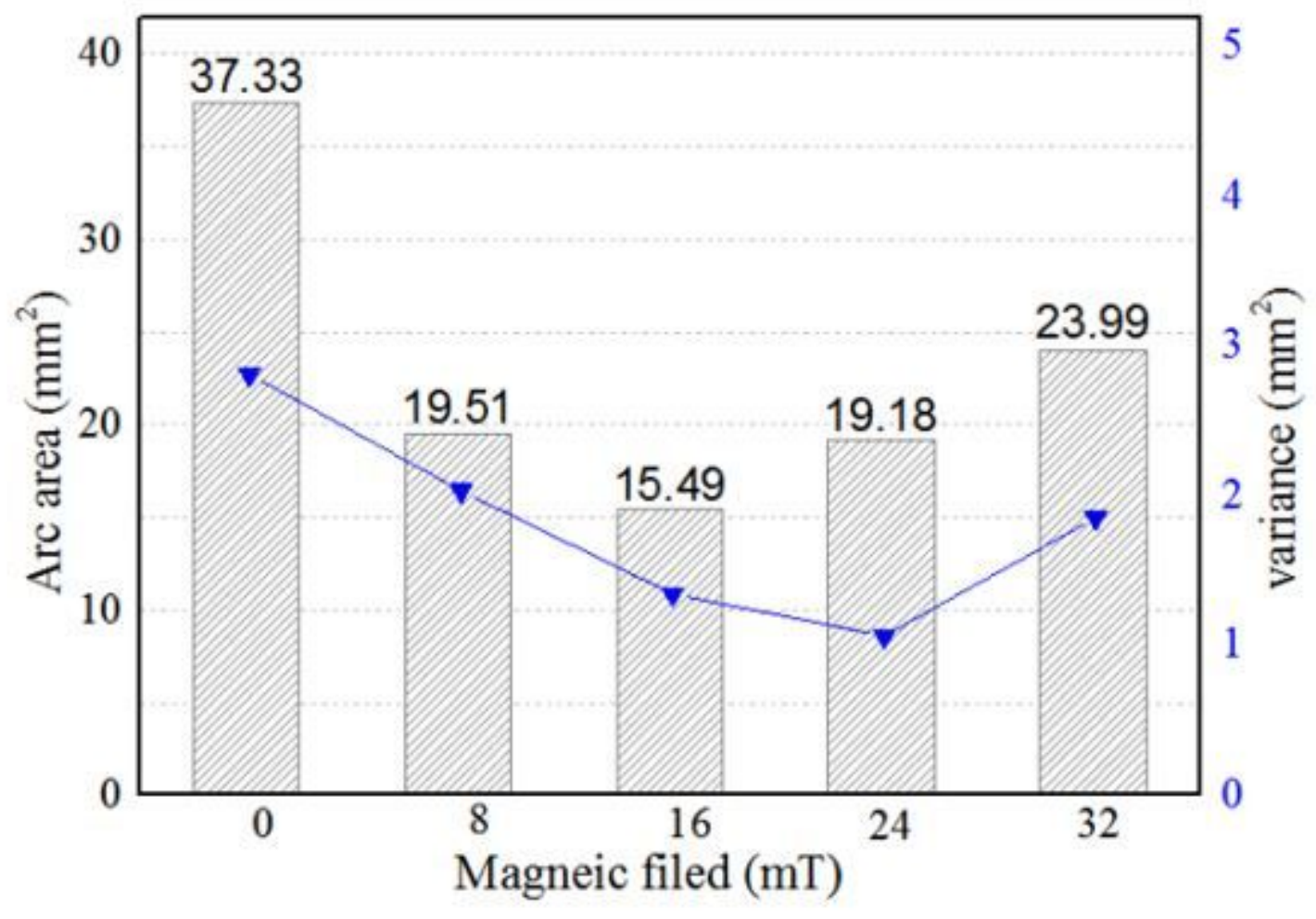

Figure 7

The maximum area and the variance of arc area under different external magnetic field

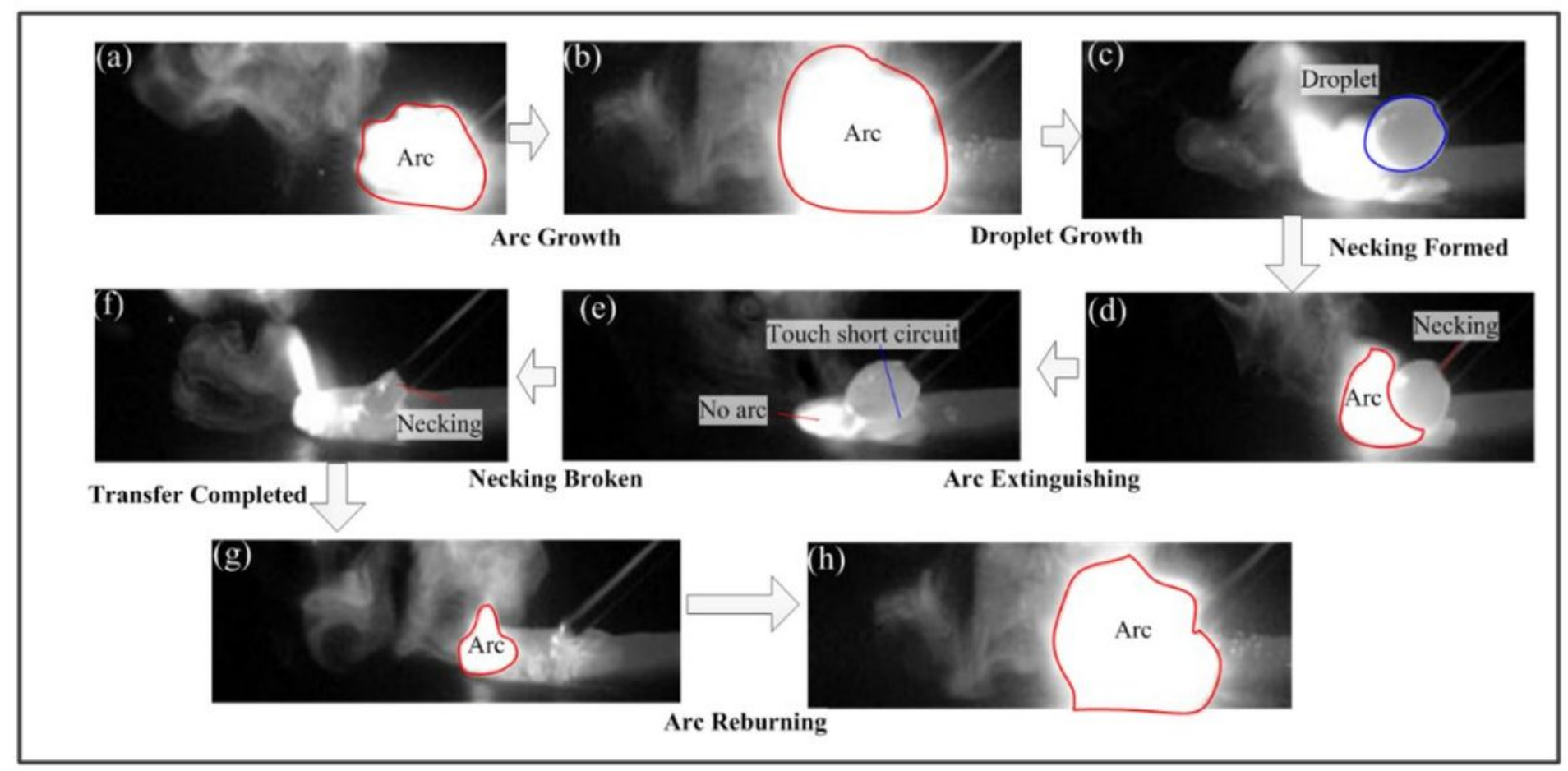


Figure 8

The droplet transfer process in Laser-MIG welding

\begin{tabular}{|c|c|c|}
\hline $\begin{array}{l}\text { Magnetic } \\
\text { field }\end{array}$ & $\begin{array}{c}\text { Droplet } \\
\text { Performance }\end{array}$ & $\begin{array}{l}\text { Average arc } \\
\text { diameter }(r)\end{array}$ \\
\hline (a) $0 \mathrm{mT}$ & & $1.8 \mathrm{~mm}$ \\
\hline (b) $8 \mathrm{mT}$ & & $1.6 \mathrm{~mm}$ \\
\hline (c) $16 \mathrm{mT}$ & & $1.2 \mathrm{~mm}$ \\
\hline (d) $24 \mathrm{mT}$ & & $1.0 \mathrm{~mm}$ \\
\hline (e) $32 \mathrm{mT}$ & & $1.1 \mathrm{~mm}$ \\
\hline Case 1 & Insufficient Droplet transfer & t transfer \\
\hline Case 3 & Exc & \\
\hline
\end{tabular}

Figure 9

Effects of different magnetic fields on droplet transfer performance 


\begin{tabular}{|c|l|l|l|}
\hline & (a) $\mathrm{MIG}$ & (b) Laser+MIG & (c) Laser+MIG+Magnetic \\
\hline $\begin{array}{c}\text { Electric } \\
\text { streamline }\end{array}$ & & & \\
\hline $\begin{array}{c}\text { Direction } \\
\mathrm{Z} \uparrow \\
\text { Electric } \\
\text { direction }\end{array}$ & Symmetrically & Towards Laser & $\begin{array}{c}\text { Rotated away } \\
\text { from Laser }\end{array}$ \\
\hline
\end{tabular}

Figure 10

Schematic of the forces acting on the pendant droplet in different welding process 


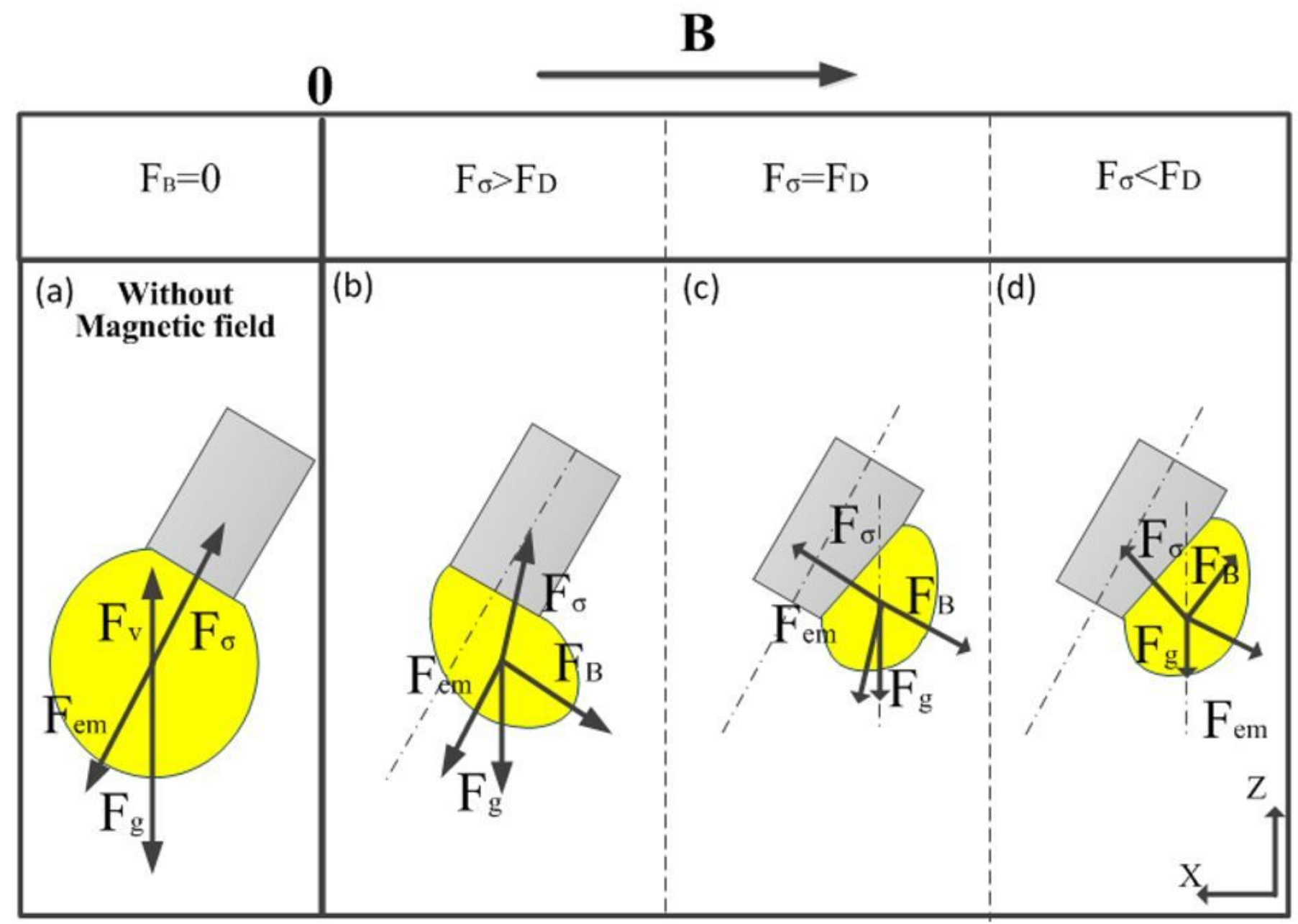

Figure 11

Main forces acting on the droplet under different external magnetic field (a) without magnetic field (b) Insufficient magnetic field (8 mT) (c) appropriate magnetic field (24 mT) (d) excessive magnetic field (32 $\mathrm{mT}$ ) 